\title{
Review of the book "Articular cartilage: Lamellar-repulsive lubrication of natural joints
}

\author{
Zenon Pawlak ${ }^{1}$ and Aleksandra Mreła ${ }^{2 *}$ \\ ${ }^{1}$ Tribochemistry Consulting, Salt Lake City, UT 84117, USA and University of Economy, Biotribology Laboratory, Garbary 2, 85-229 Bydgoszcz, PL \\ ${ }^{2}$ Kujawy and Pomorze University in Bydgoszcz, Poland
}

\section{Opinion}

The book presents a comprehensive approach to articular cartilage in joints, describing the biochemical and biophysical parameters of it and proposing the lamellar-repulsive mechanism of lubrication and it describes the mechanism of natural joint lubrication where phospholipids (PL) play the role of a lubricant. The PL physiological function in joints relies on preventing contact of bones and the degradation of the articular cartilage (AC) surface. The healthy articular cartilage causes the frictionless interaction in the joints. Then, the charge of AC is negative, and the charge of synovial fluid is positive, so the frictional coefficient is extremely low. Phospholipids from articular cartilage, called surface-active phospholipids, play an essential role of a joint lubricant during the movements of mammals. Fully understanding the joint lubrication problem is very significant during the curing the degenerated joints.

In the beginning, the book reviews the structure, composition, and function of phospholipid absorbent bilayers (BL) as a potential permanent lubricant. The original contribution of the author, focused on the interfacial energy of the PL bilayer with an emphasis on the amphoteric character of the immobile cartilage surface, is entirely original. In the joint of an animal in which the tissues slide one above the other, the mechanism of lubrication is referred to as a "lamellarrepulsive," since the AC surfaces covered with negatively charged phospholipid bilayers has got the opposite charge than synovial fluid.

In the second part, the author concentrates on the relationship between friction and wettability. Moreover, he also presents the condition of normal cartilage surface connected with the surface wettability (contact angle). During the process of air-drying, the surface of AC changes and PL bilayer transforms into the monolayer, and cartilage tissue loses its hydrophilic properties becoming hydrophobic.

In the third part, the author discusses the regeneration and renewal of degraded cartilage, concentrates on repairing the joints, especially human knees. All experimental facts, collected during friction studies, refer to wettability, surface energy, measurement of friction coefficient supports the lamellar-repulsive mechanism of natural mammal' joints.

The biotribiological model of friction in the joints, presented in the book, shows a new approach to lubrication and deserves publication. The research on the lubrication mechanism of mammal's joints is necessary for finding the methods of maintaining their health condition for many years. The comprehensive presentation of the actual state of research in the monograph is beneficial for people who want to explore this topic, especially advanced students and graduates of biomechanics and scientists who want to help people on one side and engineers who wish to build mechanical joints for robots.
In the book, Professor Zenon Pawlak gave an overview of physicochemical properties discussing cartilage surface parameters, such as wettability and surface energy. Moreover, he also developed the concept of the friction of amphoteric and negatively charged cartilage surface (-PO4-). Furthermore, the book gives a comprehensive scientific description of the friction between (AC/AC) pairs from the biomechanical, micro- and nanotribological point of view. The book can be used not only by students as a description of friction in joints, but it can also help scientists to deepen the knowledge of biological systems. People who are working in the areas of advanced technologies and nanomaterials might find useful research materials (Glimpse of Book in Figure 1) [1].

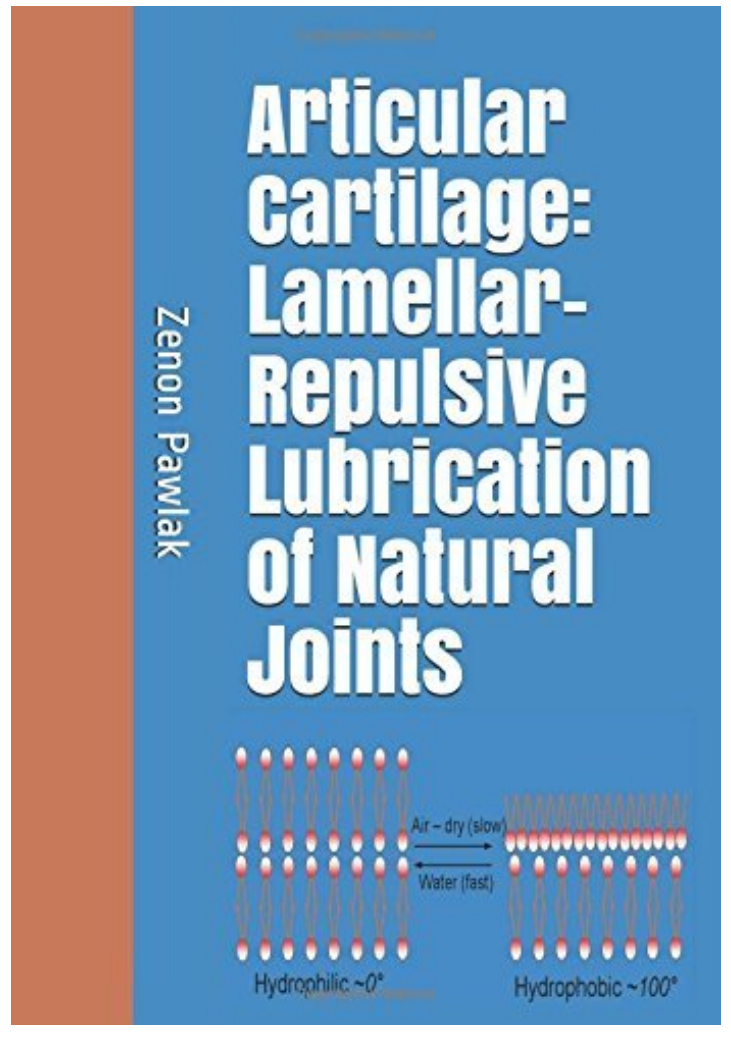

Figure 1. Cover page

${ }^{\star}$ Correspondence to: Aleksandra Mreła, Kujawy and Pomorze University in Bydgoszcz, Poland, Email: a.mrela@kpsw.edu.pl

Received: December 20, 2018; Accepted: December 28, 2018; Published: December 31, 2018 


\section{Contents}

1.0 Introduction

2.0 The structure, composition and function of cartilage

2.1 Synovial fluid

2.2 Phospholipid bilayers as a potential solid lubricant

2.3 Phospholipids

2.4 Cartilage and antiphospholipid antibody syndrome

3.0 Surface cartilage characteristics

3.1 Interfacial energy of PL bilayers

3.2 Cartilage surface wettability vs. $\mathrm{pH}$

3.3 Friction vs. wettability of cartilage surface

3.4 Amphoteric cartilage and its consequences
3.5 Cartilage surface charge density vs. $\mathrm{pH}$

4.0 Porosity of lubricated cartilage surface

5.0 The boundary and boundary-layered lubrication

6.0 Lamellar-repulsive mechanism of low friction in Nature

7.0 Resurfacing cartilage surface

8.0 Articular cartilage restoration and regeneration

9.0 Summary and outlook

About the Author

Bibliography

Index

\section{References}

1. Pawlak Z (2018), Articular Cartilage: Lamellar-Repulsive Lubrication of Natural Joints, Kindle Direct Publishing, USA 161.

Copyright: $\odot 2018$ Pawlak Z. This is an open-access article distributed under the terms of the Creative Commons Attribution License, which permits unrestricted use, distribution, and reproduction in any medium, provided the original author and source are credited. 